\title{
INVESTIGATION OF THE THERMAL SENSITIVE SPECTRAL RESPONSE OF CHALCOGENIDE FIBER BRAGG GRATING
}

This contribution presents numerical studies of the nonlinear fiber Bragg grating (FBG). The proposed model corresponds well to chalcogenide FBG as a considerable tool for optical switching. The spectral response of this device is discussed theoretically. Simulations based on the nonlinear coupled mode theory are used for investigating the relationships between the spectral transmission of nonlinear chalcogenide FBG and the grating parameters. The influence of the temperature dependence of FBG is numerically investigated. Numerical results show that the ambient temperature has an influence on the spectral response of FBG. The results are indications for applications in optical switches for all-optical communication networks and this can be important for wavelength division multiplexing optical networks.

Keywords: Chalcogenide glass, fiber Bragg gratings, optical switching, thermal sensitivity, spectral response.

\section{Introduction}

Optical bistable devices have a large number of applications in all-optical communication systems. They are building blocks of optical logic, memories, switches, light power limiters etc. that are crucial parts of optical network. In this paper we focused on the optical switches devices. Ultrafast optical switches are necessary parts of optical network nodes in high bit rate all-optical communication networks including systems of wavelength division multiplexing (WDM) [1, 2]. The phenomenon of optical bistability (OB) is important to realize nonlinear switching in FBG at a desired wavelength. This optical phenomenon refers to the situation in which an optical device exhibits two stable different output intensities under one given incident intensity. $\mathrm{OB}$ arises from the energy exchange between light and nonlinear optical medium. It arises in feedback structures with some loss or nonlinearity [3, 4].

Nonlinear chalcogenide glasses as the nonlinear switching medium have advantages over other materials including ultrafast response time [5]. Chalcogenide glasses (CGs) are based on the chalcogen elements $\mathrm{S}, \mathrm{Se}$ and Te with the addition of other elements such as $\mathrm{Ge}, \mathrm{As}$ and $\mathrm{Sb}$ to form stable glasses. CGs are low-phonon energy materials and are generally transparent from near-infrared to mid-infrared. These glasses are well-known as optical materials with high nonlinear optical properties. High third-order Kerr nonlinearities up to 1000 times higher than those of silica glass make them distinguished candidates for applications such as nonlinear switching, Raman amplification, optical regeneration, parametric amplification and supercontinuum generation [5-7].

FBG is an important component of wavelength division multiplexed (WDM) optical communication systems $[8,9]$. But there is still the problem of variable optical communication path characteristics and environmental fluctuations [10,11].We investigate the nonlinear chalcogenide fiber Bragg gratings (FBG) as a promising device for all-optical switching. This effect can be modified depending on the incident light power which is assumed to be due to the optical Kerr effect. The aim of this contribution is to examine the bistable behavior and the spectral characteristics in a nonlinear chalcogenide FBG under the influence of the ambient temperature theoretically. The incident intensity thresholds of the bistable regimes are analyzed numerically to deduce possible switching properties of FBGs based on one chalcogenide glass sample taken from [12]. Nonlinear coupled mode equations (NCMEs) are used to model forward and backward light waves in nonlinear chalcogenide FBG. As the thermo-optic changes of the refractive index usually dominate over the changes due to the fiber elongation, only the thermo-optic effect on the refractive index is considered here.

\section{Background}

The nonlinear effect available in the third-order Kerr nonlinear media results in the intensity dependent refractive index change, which causes an intensity dependent phase modulation. When this phase modulation is converted into an amplitude modulation the switching can result [13].

\section{A Fiber Bragg Grating}

FBG (Fig. 1) is fabricated by exposing an optical photosensitive fiber to a pattern of UV light. This causes the changes of the refractive index along the FBG length $L$ periodically. In Fig. $1 I_{\text {in }}$ represents the incident light intensity, $I_{\text {ref }}$ the reflected intensity and $I_{\text {tran }}$ the transmitted intensity. The transmittance is given as

\footnotetext{
* Eliska Jurisova, Libor Ladanyi, Jarmila Mullerova

Institute of Aurel Stodola Liptovsky Mikulas, Faculty of Electrical Engineering, University of Zilina, Slovakia, E-mail: jurisova@1m.uniza.sk
} 
the ratio between $I_{\text {tran }}$ and $I_{i n}$ as $T=I_{\text {tran }} / I_{i n}$. The key property of gratings is that they exhibit the Bragg reflection around a designed wavelength, the so-called Bragg wavelength $\lambda_{\text {Bragg }}$. This corresponds to the central rejection peak in the transmittance given by the Bragg condition [13, 14]

$$
\lambda_{\text {Bragg }}=2 n_{\text {eff }} \Lambda
$$

where $n_{\text {eff }}$ is the effective index of the fiber core and $\Lambda$ is the grating spatial period.

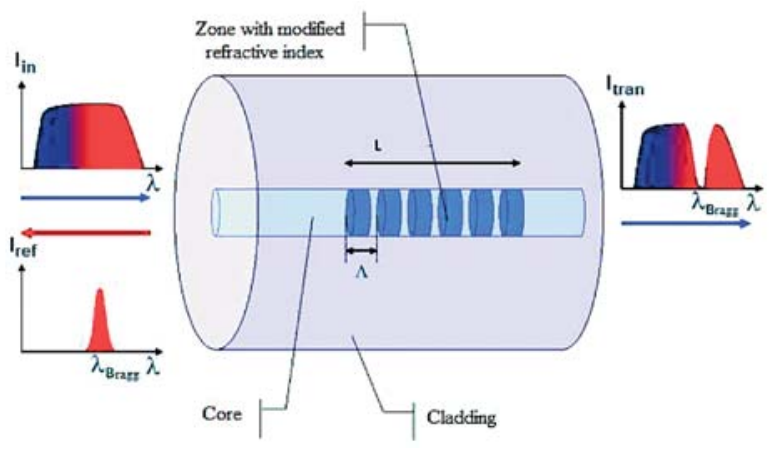

Fig.1 Design of propagating waves in the fiber Bragg grating [15]

FBG consists of a material, the optical parameters of which could be affected by heat. As the FBG temperature changes, a wavelength change defined by the shift of $\Delta \lambda_{B r a g g}$ from the original wavelength $\lambda_{\text {Bragg }}$ can be observed. This change is given by

$$
\Delta \lambda_{\text {Bragg }}=\lambda_{B O}\left(\alpha_{e}+\alpha_{o}\right) \Delta T_{F B G}
$$

where $\lambda_{B O}$ is the FBG Bragg wavelength at a reference temperature of $T_{R}, \alpha_{e}$ is the thermal expansion coefficient of the fiber, $\alpha_{o}$ is the thermo-optic coefficient. $\Delta T_{F B G}=T_{A}-T_{R}, T_{A}$ is the ambient temperature. The FBG thermal sensitivity $S_{F B G}$ is defined as $S_{F B G}=$ $=\Delta \lambda_{B r a g g} / \Delta T_{F B G}$. Hence, the thermally influenced Bragg wavelength is defined as [16]

$$
\lambda_{B r a g g}=\lambda_{B O}+S_{F B G} \Delta T_{F B G}
$$

The refractive index inside the fiber core varies along the grating length periodically. The index variation can be expressed as $[15,17]$

$$
n=n_{e f f}+V_{n} \nu \cos \left(\frac{2 \pi z}{\Lambda}\right)+n_{2}|E|^{2}
$$

Here $V_{n}$ is the depth of the modulation, $v$ the fringe visibility, $n_{2}$ is the nonlinear index of optical fiber, $E$ is the electric field amplitude. The $z$ coordinate denotes the grating direction. The electric field inside the grating can be written as

$$
E=\left[F e^{\left(i k_{B} z\right)}+B e^{\left(-i k_{B} z\right)}\right] e^{\left(-i \omega_{0} t\right)}
$$

where $k_{B}=\pi / \Lambda n_{\text {eff }}$ is the wavenumber in the medium at the Bragg resonance, $F$ and $B$ are the amplitudes of the transverse core mode fields of the forward and backward (counter-propagating) waves, respectively. $F$ and $B$ exhibit additional slow variations as functions of position $z$ and time $t$; they are thus the envelope functions. The carrier optical frequency at which the wave spectrum is initially centered is marked as $\omega_{0}$. We assume that $|E|^{2} \sim I$ represents the intensity of light.

\section{$B$ Nonlinear coupled mode theory}

The forward and backward light waves exist simultaneously and satisfy NCMEs [17-19]:

$$
\begin{aligned}
& i \frac{\partial F}{\partial z}+i \frac{1}{v_{g}} \frac{\partial F}{\partial t}+\kappa B+\delta F+ \\
& +\gamma|F|^{2} F+2 \gamma|B|^{2} F=0 \\
& -i \frac{\partial B}{\partial z}+i \frac{1}{v_{g}} \frac{\partial B}{\partial t}+\kappa F+\delta B+ \\
& +\gamma|B|^{2} B+2 \gamma|F|^{2} B=0
\end{aligned}
$$

where the variable $v_{g}=c / n_{\text {eff }}$ represents the group velocity in the absence of the grating. The parameter $\kappa$ is the coupling coefficient representing the grating strength per unit length. It determines the energy ratio between the forward and the backward fields and is defined as

$$
\kappa=\frac{\pi V_{n}}{\lambda_{\text {Bragg }}}
$$

The detuning parameter $\delta$ is the relation between the Bragg wavelength $\left(\lambda_{\text {Bragg }}\right)$ and the carrier wavelength $(\lambda)$ given as

$$
\delta=2 \pi n_{\text {eff }}\left(\frac{1}{\lambda}-\frac{1}{\lambda_{\text {Bragg }}}\right)
$$

The variable $\gamma$ symbolizes the nonlinear (Kerr) coefficient (if $\gamma=0$ the linear regime occurs)

$$
\gamma=\frac{2 \pi n_{2}}{\lambda_{\text {Bragg }}}
$$

This parameter enters the term $\gamma \mid F^{2} F$ (or $\gamma|B|^{2} B$ ) in (6) (or 7 ) determining the strength of the self-phase modulation. The last term in (6) (or 7) belongs to the cross-phase modulation.

The boundary conditions in the spatial domain are usually chosen at the two opposite sides of the grating. The initial and boundary conditions take the well-known form [17-19]

$$
\begin{aligned}
& F=(z=0, t)=A(t) ; B=(z=L, t)=0 ; \\
& F(z, 0)=0 ; B(z, 0)=0
\end{aligned}
$$

The second of these equations indicates that the input wave is launched only from the $z=0$ position. In this paper the finite difference method (FDM) was used to solve NCMEs (6), (7).

\section{Optical bistability}

$\mathrm{OB}$ is a promising way to realize an optical switch, a key component of integrated photonic devices. The Kerr (dispersive) optical bistability is based on coupling the feedback mechanism in a non- 
linear optical medium that exhibits a change in the refractive index as a function of incident light intensity. Fig. 2 shows the shape of the curve representing OB as the following change of the transmittance $(T)$ as a function of the incident intensity $\left(I_{i n}\right)$.

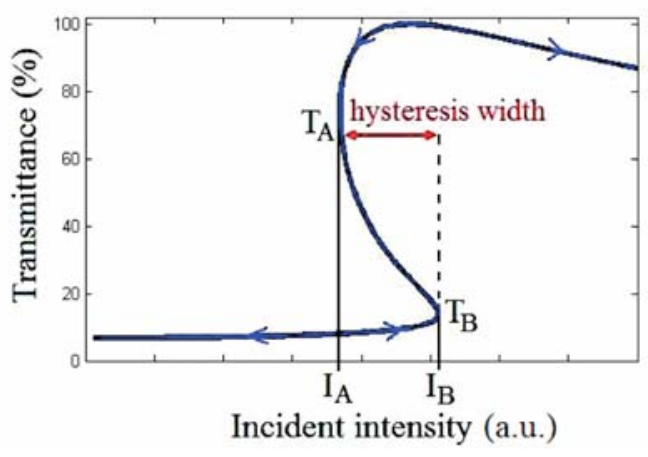

Fig. 2 The optical bistability curve

While $I_{\text {in }}$ is pumped slightly the system behaves as linear until reaching the point $I_{B}$ (the switch-on threshold). Here the output intensity makes a sudden jump to the upper branch of the hysteresis loop. When the optical intensity decreases from high values, it can return to the lower branch at the switch-off threshold $\left(I_{A}\right.$, Fig. 2).The width of the hysteresis loop is defined as the difference between the switch-on $\left(I_{B}\right)$ and -off $\left(I_{A}\right)$ thresholds. Let us call the state of a linear increase of $I_{i n}$ as the "OFF" state and the state of a sharp rise in $I_{i n}$ for $I_{i n}>I_{A}$ as the "ON" state. The transmission difference between "ON" and "OFF" states is the so-called on-off contrast. The width of the S-shaped curve (the so-called hysteresis width, $w$ ) is the difference between the switch-on (point $I_{B}$ ) and switch-off (point $I_{A}$ ) intensities. The middle branch of the S-curve is unstable against amplitude fluctuations due to the negative slope of the transmittance curve.

\section{Results and numerical simulations}

Here we present the simulation results and discuss the bistable behavior and the threshold of optical switching intensities in nonlinear chalcogenide FBGs. Then, the spectral responses of nonlinear FGBs at different incident intensities and at varied temperatures are numerically investigated. The experimental data necessary for numerical studies were taken from $[12,20]$. The parameters of $\mathrm{As}_{2} \mathrm{Se}_{3}$ chalcogenide used in the simulations are in Table 1.

We investigated $\mathrm{OB}$ in the $3^{\text {rd }}$ transmission spectral window of the optical communication WDM systems. The Bragg wavelength was positioned at the wavelengths of $\lambda_{\text {Bragg } 1}=1550.12 \mathrm{~nm}$ and $\lambda_{\text {Bragg } 2}=1552.52 \mathrm{~nm}$ representing telecommunication C-band channels of the $100 \mathrm{GHz}$ and $50 \mathrm{GHz}$ channel spacing grid according to G.694 ITU-T standard. The neighboring channel separation in the vicinity of $1550 \mathrm{~nm}$ for the $100 \mathrm{GHz}(50 \mathrm{GHz})$ channel spacing is $0.8 \mathrm{~nm}(0.4 \mathrm{~nm})$. In our investigation we assume
Parameters of FBG and CG taken from literature $[12,20]$ Table 1

\begin{tabular}{|c|c|c|}
\hline Grating length & $L$ & $1 \mathrm{~cm}$ \\
\hline Depth of the modulation & $V_{n}$ & $1 \times 10^{-4}$ \\
\hline Bragg wavelength & $\lambda_{\text {Braggl }}$ & $1550.12 \mathrm{~nm}$ \\
\hline Bragg wavelength & $\lambda_{\text {Bragg } 2}$ & $1552.52 \mathrm{~nm}$ \\
\hline Carrier wavelength & $\lambda$ & $\left(\lambda_{\text {Bragg }} \pm 0.2\right) \mathrm{nm}$ \\
\hline Fringe visibility & $v$ & 1 \\
\hline Effective index $\mathrm{As}_{2} \mathrm{Se}_{3}$ & $n_{e f f}$ & 2.81 \\
\hline Nonlinear index As $\mathrm{Se}_{3}$ & $n_{2}$ & $14 \times 10^{-14} \mathrm{~cm}^{2} / \mathrm{W}$ \\
\hline Reference temperature & $T_{R}$ & $10\left[{ }^{\circ} \mathrm{C}\right]$ \\
\hline Ambient temperature & $T_{A}$ & $<8,15>\left[{ }^{\circ} \mathrm{C}\right]$ \\
\hline Thermal expansion coefficient & $a_{e}$ & $4.10^{-6}\left[\mathrm{~K}^{-1}\right]$ \\
\hline Thermo-optic coefficient & $a_{o}$ & $21.10^{-6}\left[\mathrm{~K}^{-1}\right]$ \\
\hline
\end{tabular}

$50 \mathrm{GHz}$ channel spacing. We suppose the stationary behavior of the nonlinear system. Then NCMEs (6) and (7) have the form

$$
\begin{aligned}
& \frac{\partial F}{\partial z}=i\left[\kappa B+\delta F+\gamma\left(|F|^{2}+2|B|^{2}\right) F\right] \\
& \frac{\partial B}{\partial z}=-i\left[\kappa F+\delta B+\gamma\left(|B|^{2}+2|F|^{2}\right) B\right]
\end{aligned}
$$

\section{A Transmittance versus incident intensity}

Fig. 3 shows the nonlinear characteristics for $\mathrm{As}_{2} \mathrm{Se}_{3}$. The detuning parameter $\delta=0$, that means $\lambda=\lambda_{\text {Bragg }}$. We observe the change in the transmittance: when the incident intensity increases from zero, the transmittance traces the lower branch of the line until the input intensity achieves the switching point, where the output makes a rapid jump to the upper branch. From this figure it is clear that the change in the Bragg wavelength does not cause a considerable change in the bistable curves. The S-shapes, the transmittances, switching intensities, the hysteresis widths and the on-off contrasts are very similar.

\section{$B$ Investigation of spectral responses}

The possible distortions of spectral responses may occur in the OB incident intensity regions. Figs. 4 and 5 show the transmittance spectra of one samle of nonlinear chalcogenide FBG with the incident intensity as a parameter. The transmittance spectrum of linear FBGs is shown for the comparison (the dashed line). In the linear regime $(\gamma=0)$, the grating response does not depend on the incident intensity. As seen from the figures, the spectral curve is symmetrical on both sides of $\lambda_{\text {Bragg }}$ at which a pronounced central transmittance minimum occurs. The simulations were carried out for changing the difference $\Delta \lambda=\lambda-\lambda_{\text {Bragg }}$ of the carrier wavelength $\lambda$ and the Bragg wavelength $\lambda_{\text {Bragg }}$ from $\Delta \lambda=-0.2 \mathrm{~nm}(\lambda=$ $=1552.32 \mathrm{~nm})$ up to $\Delta \lambda=0.2 \mathrm{~nm}(\lambda=1552.72 \mathrm{~nm})$. Two cases for the same sample of chalcogenide nonlinear FBG were studied in more details: the incident intensity $I_{\text {in }}=100 \mathrm{MW} / \mathrm{cm}^{2}$ (Fig. 4) and $I_{\text {in }}=230 \mathrm{MW} / \mathrm{cm}^{2}$ (Fig. 5). 
At the incident intensity of $100 \mathrm{MW} / \mathrm{cm}^{2}$ the transmittance is very low and the system behaves almost as linear. However from Fig. 4 we can clearly see that nonlinear variation of the refractive index shifts the transmittance spectrum toward longer wavelengths and the change of the shape of the spectral curve (SC) occurs. We observe the SC narrowing for wavelengths below but near to $\lambda_{B r a g g}$ $(\Delta \lambda<0)$ and enlarging for wavelengths above $\lambda_{\text {Bragg }}(\Delta \lambda>0)$. The side lobes are shifted to higher $\Delta \lambda$.

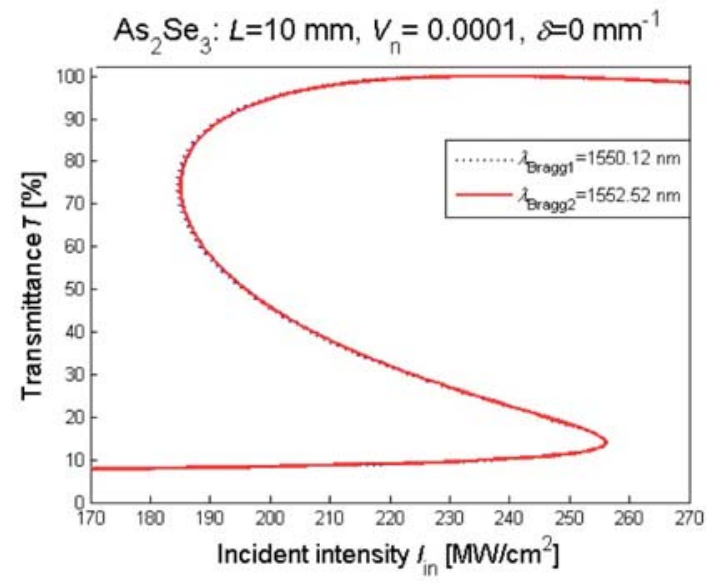

Fig. 3 FBG transmittance versus the incident intensity calculated as the time-independent solutions of NCMEs. The comparison of the optical bistability curves for chalcogenide FBG at the Bragg wavelengths of

$$
\lambda_{\text {Bragg } 1}=1550.12 \text { and } \lambda_{\text {Bragg } 2}=1552.52
$$

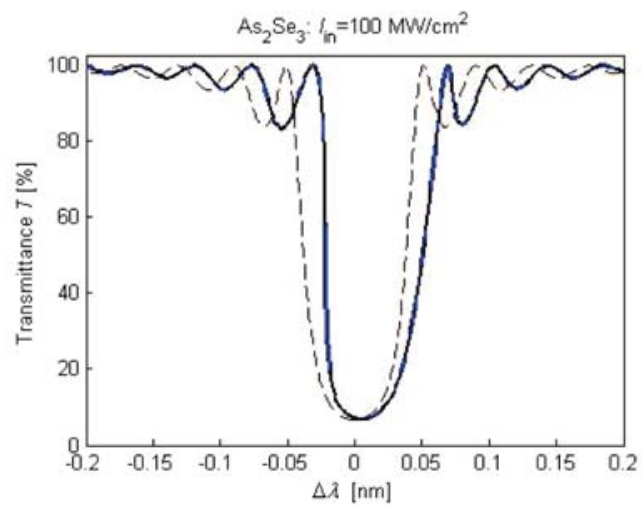

Fig. 4 Transmittance spectra of the $\mathrm{As}_{2} \mathrm{Se}_{3}$ nonlinear FBG at the incident intensity of $100 \mathrm{MW} / \mathrm{cm}^{2}$ (solid line) and for the linear FBG of the same parameters (the dashed line)

The incident intensity of $230 \mathrm{MW} / \mathrm{cm}^{2}$ corresponds to the case when two transmittance states are possible for the same incident intensity. Fig. 5a) corresponds to the "ON" state, Fig. 5b) to the "OFF" state. It is obvious that the nonlinearity causes the dis- tortion of the spectrum in the "ON" state and the distortion and the shift toward higher $\Delta \lambda$ in the "OFF" state. The spectral change between "ON" and "OFF" states was found to be $\Delta \lambda \sim 0.019 \mathrm{~nm}$ that is smaller than the WDM channel spacing. Therefore, no problems with the undesirable channel switching between "ON" and "OFF" states are expected.

C Investigation of spectral responses by the temperature influence

Here we investigate the impact of ambient temperature on the nonlinear chalcogenide FBG spectrum. The same sample parameters as in Table 1 were used. Figure 6 shows the OB curve for five different values of the ambient temperature. We observe that the temperature change has a great influence on the curve $\mathrm{OB}$ and the width of the hysteresis of on - off contrast. In Fig. 7 the bistable behavior for the input intensity of $255 \mathrm{MW} / \mathrm{cm}^{2}$ was simulated at "ON" and "OFF" state at varied ambient temperatures.

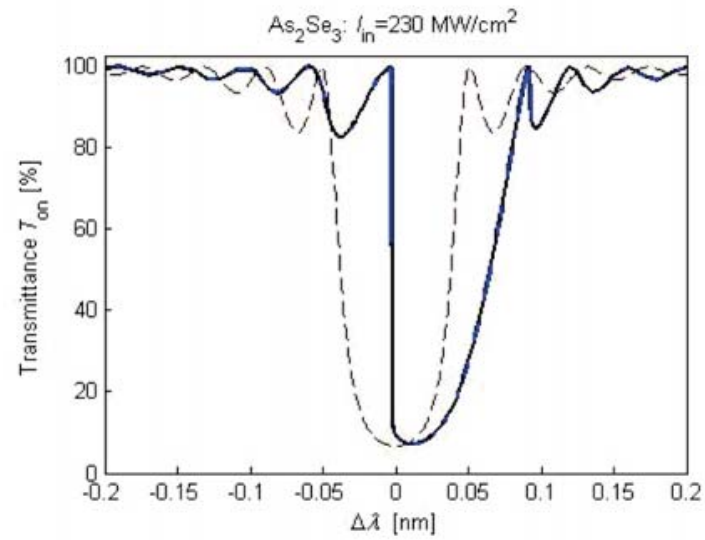

a) "ON" state

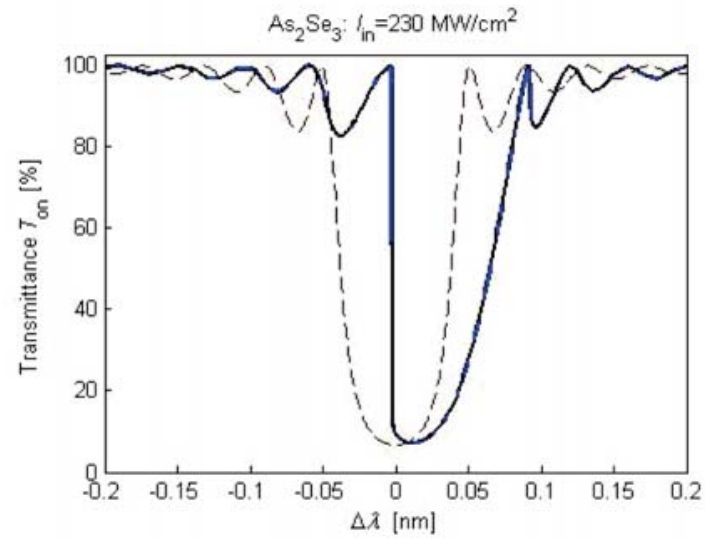

b) “OFF" state

Fig. 5 Transmittance spectra in the "ON" (a)) and "OFF"(b)) states at the incident intensity of $230 \mathrm{MW} / \mathrm{cm}^{2}$ (solid line) and for the linear $F B G$ of the same parameters (the dashed line) 


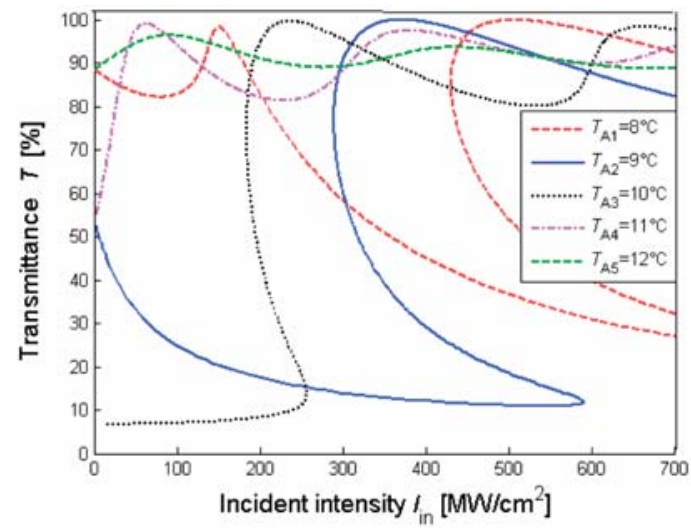

a)

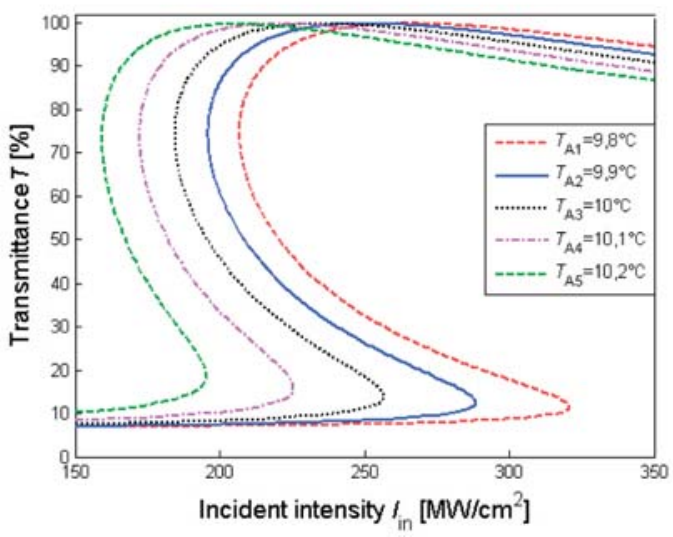

b)

Fig. 6 FBG spectrum at varied ambient temperature a) $T_{A}$ in the range from $8{ }^{\circ} \mathrm{C}$ to $12{ }^{\circ} \mathrm{C}$ and $\left.b\right) T_{A}$ in a narrow temperature range from $9.8^{\circ} \mathrm{C}$ to $10.2^{\circ} \mathrm{C}$

From Fig. 7 we conclude that spectral curves do not change in shapes, but the shift occurs. If the temperature is increased by $5{ }^{\circ} \mathrm{C}$, there is a shift of $\sim 0.186 \mathrm{~nm}$. The variation of approx. $12{ }^{\circ} \mathrm{C}$ shifts the spectrum by more than $0.4 \mathrm{~nm}$. This value corresponds to the spacing of the $50 \mathrm{GHz}$ WDM grid in the vicinity of the $1550 \mathrm{~nm}$ wavelength. Then the FBG temperature change may affect FBG spectrum and channel switching in WDM systems may occur.

\section{Conclusion}

In this article, optical switching based on optical bistability in nonlinear chalcogenide FBG is investigated. The sample of $\mathrm{As}_{2} \mathrm{Se}_{3}$ is numerically studied as a FBG medium. The nonlinear coupled mode equations are applied to analyze the optical bistable behavior.
Numerical results are gathered using the finite difference method. The shape of the hysteresis loop is considered from the point of view of optical switching. The influence of the detuning of the carrier and the Bragg wavelength on the optical bistability behavior and the threshold of the incident switching intensity is showed. Spectral responses of the investigated FBGs are numerically studied and shown to be distorted at some investigated input intensities causing the nonlinearities. We found that the temperature change the linear shift in the spectrum of wavelengths that can affect switching channels in the WDM systems.

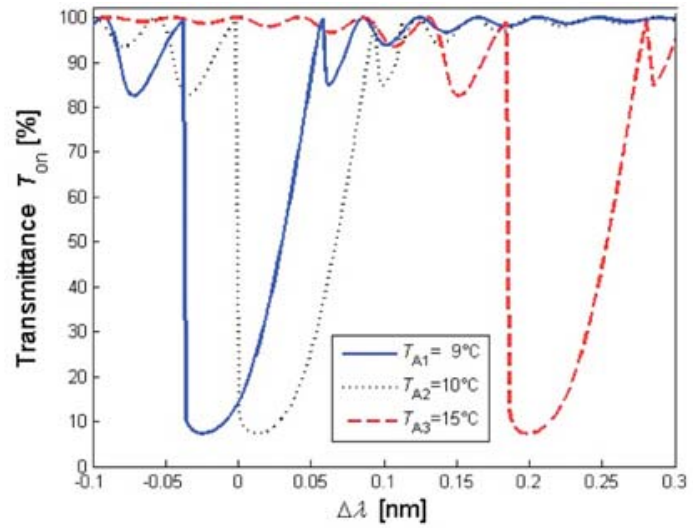

a)

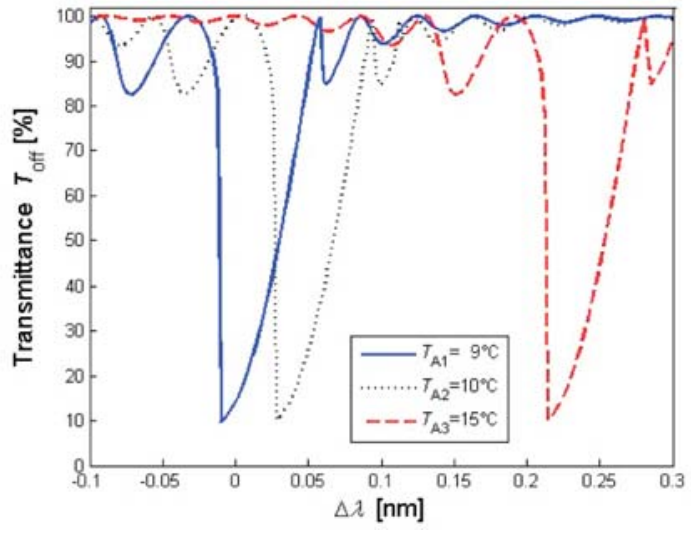

b)

Fig. 7 FBG spectrum at varied temperatures, a) "ON" state and b) "OFF" state

\section{Acknowledgment}

This work was partly supported by the Slovak Grant Agency under the project No. 1/1271/12. 


\section{References}

[1] DADO, M., KRAJCI, S., DUBOVAN, J., SABOL, D.: Numerical Investigation of Optical Burst Switching. Communication - Scientific Letters of the University of Zilina, No. 2, pp. 20-24, 2008

[2] HENKER, R. et al.: A Review of Slow- and Fast- Light Based on Stimulated Brillouin Scattering in Future Optical Communication Networks. Communication - Scientific Letters of the University of Zilina, pp. 45-52, No. 4, 2008

[3] WINFUL, H. G., MARBURGER, S. D., GARMIRE, E.: Theory of Bistability in Nonlinear Distributed Feedback Structures, Appl. Phys. Lett., 35 (5), 1979

[4] YOSIA, Y., PING, S.: Optical Bistability in Periodic Media with Third-, Fifth-, and Seventh-Order Nonlinearities, J. Lightwave Technol., vol. 25, pp. 3875-3882, 2007

[5] HARBOLD, J. M.,ILDAY, F. O., WISE, F. W. and AITKEN, B. G.: Highly Nonlinear Ge-As-Se and Ge-As-S-Se Glasses for AllOptical Switching, IEEE Photonics Technology Letters, vol. 14, No. 6, pp. 822-824, June 2002

[6] SANGHERA, G. J. S. et al.: Nonlinear Properties of Chalcogenide Glass Fibers, Optoelectron. Adv. M, vol. 8, No. 6, pp. $2148-2155,2006$

[7] ZAKERY, A., ELLIOT, S. R.: Optical Nonlinearities in Chalcogenide Glasses and their Applications, Springer-Verlag Berlin Heidelberg, 2007

[8] ERDOGAN, T.: Fiber Grating Spectra, J. of Lightwave Technology, vol. 15, No. 8, 1997

[9] LI, W., Y.W. LI, X.D. HAN, G.Q. YU: The Study of Enhancing Temperature Sensitivity for FBG Temperature Sensor, $8^{\text {th }}$ Intern. Conference on Machine Learning and Cybernetics, Baoding, 2007

[10] MAHMOUD, M. and GHASSEMLOOY, Z.: Tunable Fiber Bragg Gratings Modeling and Simulation, IEEE, Computer Society, 2003

[11] KAMIKAWACHI, R.C., KALINOWSKI, H.J., MULLER, M., FABRIS, J.L.: Thermal Behaviour of an Etched FBG Immersed in Different Surroundings. J. of Microwaves, Optoelectronics and Electromagnetic Applications, vol. 6, No. 2., 2007

[12] BOUDEBS, G., et. al.: Nonlinear Optical Properties of Chalcogenide Glasses at Telecommunication Wavelength Using Nonlinear Imaging Technique, Transparent Optical Networks, vol. 2, pp. 145-150, 2004

[13] IMAI, M., SATO, S.: Optical Switching Devices Using Nonlinear Fiber-Optic Grating Coupler, Photonics Based on Wavelength Integration and manipulation, IPAP Books 2, pp. 293-302, 2005

[14] CARVALHO, J.C.C. et al.: A New Acceleration Technique for the Design of Fiber Gratings, Optical Society of America, Optic Express, vol. 14, No. 22, pp.10715-10725, 2006

[15] MISSINNE, J., HOE, B.: Artificial Skin Based on Flexible Optical Tactile Sensors, SPIE Newsroom, DOI: 0.1117/ 2.1201001.002582, 2010

[16] MAHMOUD, M., GHASSEMLOOY, Z: Tunable Fiber Bragg Gratings Modeling and Simulation. IEEE, Computer Society, 2003

[17] PENG, Y., QIU, K.,WU, B., JI, S.: Study on Characteristics of Optical Bistable Devices Based on Fiber Bragg Grating, Passive Components and Fiber-based Devices VI, SPIE-OSA-IEEE, Proc. SPIE, vol. 7630, 2009

[18] LEE, H., AGRAWAL, G. P: Nonlinear Switching of Optical Pulses in Fiber Bragg Gratings, IEEE J. of Quantum Electronics, vol. 39, No. 3, 2003

[19] STERKE, C.M., JACKSON, K.R., ROBERT, B.D.: Nonlinear Coupled Mode Equations on a Finite Interval: a Numerical Procedure, J. Opt. Soc. Am B, vol. 8, No. 2, 403-412, 1991

[20] GUILLEVIC, E. et al.: Optimatization of Chalcogenide Glass in the As-Se-S System for Automative Applications. OPT MATER, pp. 1688-1692, vol. 31, No. 11., 2009. 